\title{
MicroRNA profiling reveals that miR-21, miR486 and miR-214 are upregulated and involved in cell survival in Sézary syndrome
}

\author{
MG Narducci ${ }^{1}$, D Arcelli ${ }^{1}$, MC Picchio ${ }^{1}$, C Lazzeri ${ }^{1}$, E Pagani ${ }^{1}$, F Sampogna ${ }^{1}$, E Scala ${ }^{1}$, P Fadda ${ }^{1}$, C Cristofoletti ${ }^{1}$, A Facchiano ${ }^{1}$,
} M Frontani ${ }^{1}$, A Monopoli ${ }^{1}$, M Ferracin ${ }^{2}$, M Negrini ${ }^{2}$, GA Lombardo ${ }^{1}$, E Caprini ${ }^{1}$ and G Russo ${ }^{\star, 1}$

Sézary syndrome (SS) is an incurable leukemic variant of cutaneous T-cell lymphoma and its pathogenesis is still unknown. Diagnosis/prognosis may strongly ameliorate the management of SS individuals. Here, we profiled the expression of 470 microRNAs (miRNAs) in a cohort of 22 SS patients, and we identified 45 miRNAs differentially expressed between SS and controls. Using predictive analysis, a list of 19 miRNAs, including miR-21, miR-214, miR-486, miR-18a, miR-342, miR-31 and let-7 members were also found. Moreover, we defined a signature of 14 miRNAs including again miR-21, potentially able to discriminate patients with unfavorable and favorable outcome. We validated our data for miR-21, miR-214 and miR-486 by qRTPCR, including an additional set of array-independent SS cases. In addition, we also provide an in vitro evidence for a contribution of miR-214, miR-486 and miR-21 to apoptotic resistance of CTCL cell line.

Cell Death and Disease (2011) 2, e151; doi:10.1038/cddis.2011.32; published online 28 April 2011

Subject Category: Cancer

Sézary syndrome (SS) is a rare and aggressive leukemic variant of cutaneous T-cell lymphoma (CTCL) characterized by the presence of neoplastic lymphocytes named Sézary cells, in the skin, lymph nodes and peripheral blood. ${ }^{1}$ Consistently with the complex phenotype observed, SS is associated with a poor clinical outcome with an estimated 5 -years survival of only $25 \% .{ }^{2}$ Until now, no specific therapy is available for this disease. ${ }^{3}$ Although numerous efforts have been made to identify pathogenically relevant genes, the cause of SS is still unknown. With this aim, high throughput analyses have been recently done on whole genome and transcriptome of CTCL identifying regions of recurrent allelic imbalance and altered profiles of gene expression. $^{4-7}$

miRNAs control gene expression at the posttranscriptional level by causing mRNA degradation and/or repressing mRNA translation. They have a wide recognized role in development, differentiation, growth, apoptosis and stress responses, as well as in T-cell homeostasis. ${ }^{8-11}$ A growing number of studies indicate that deregulated miRNAs have a key role in cancer development, acting both as oncogenes and as tumor suppressor genes. This evidence is also supported by the fact that more than $50 \%$ of miRNA-encoding loci reside in chromosomal regions unbalanced during tumorigenesis. Furthermore, miRNA expression profiles are able to predict disease status and clinical outcome, as well as tumor progression and therapeutic responses. ${ }^{12}$

Expression patterns of miRNAs and their role in the pathogenesis of SS have only recently been addressed. ${ }^{13}$
Here, we present a miRNA profile of 22 SS patients identifying a signature that distinguishes malignant from healthy lymphocytes, as well as a series of miRNAs associated with disease and clinical outcome, and an in vitro evidence for a contribution of miR-214, miR-486 and miR-21 to apoptotic resistance of CTCL cell line.

\section{Results}

Differential miRNA expression between SS and normal T cells. To identify miRNA signature in SS, we profiled the expression of 28 SS samples (22 patients and 6 follow-up) and six healthy controls (HCs) by the means of microarray analysis containing 470 human miRNAs. To determine whether global miRNA profiling could distinguish molecular groups, we performed an unsupervised analysis using heat map package of bioconductor. As showed by dendrogram (Figure 1a and Supplementary Figure S1) miRNAs separated the HCs from SS samples. In particular, HCs $(n=6)$ were clearly grouped and statistically validated in a distinct sub-branch, whereas SS patients were sub-divided in four distinct clusters (correlation $=0.95$ ). The first one, very close to normal controls, contained 4 SS samples and the other three clusters comprised 9, 6 and 9 SS samples, respectively. Four of six follow-up (mSS43_1, 30_1, 39_1 and 33_1) were clustered together with the respective original specimen, whereas mSS38_1 and 38_2 clustered in a different group, indicating that a change might have

${ }^{1}$ Istituto Dermopatico dell'Immacolata-IRCCS, Roma, Italy and ${ }^{2}$ Dipartimento di Medicina Sperimentale e Diagnostica, Università di Ferrara, Ferrara, Italy *Corresponding author: G Russo, IDI-IRCCS, Via dei Monti di Creta 104, 00167 Rome, Italy. Tel: + 390666462432; Fax: + 390666462430; E-mail: russo@ idi.it Keywords: Sézary syndrome; microRNAs; diagnostic and prognostic markers

Abbreviations: SS, Sézary syndrome; CTCL, cutaneous T cell lymphoma; miR, microRNA; BRB, biometric research branch; KM, Kaplan-Meier; PBMCs, peripheral blood monuclear cells; qRT-PCR, quantitative-real time-PCR; FC, fold-change; LNA, locked nucleic acid; MTT, 3-(4,5-dimethylthiazol-2-yl)-2,5-diphenyltetrazolium bromide; FACS, fluorescence activated cell sorting; PI, propidium iodide; NFYC, nuclear transcription factor Y, gamma; EVL, Enah/Vasp-like; PTEN, phosphatase and tensin homolog

Received 03.3.11; accepted 15.3.11; Edited by P Salomoni 

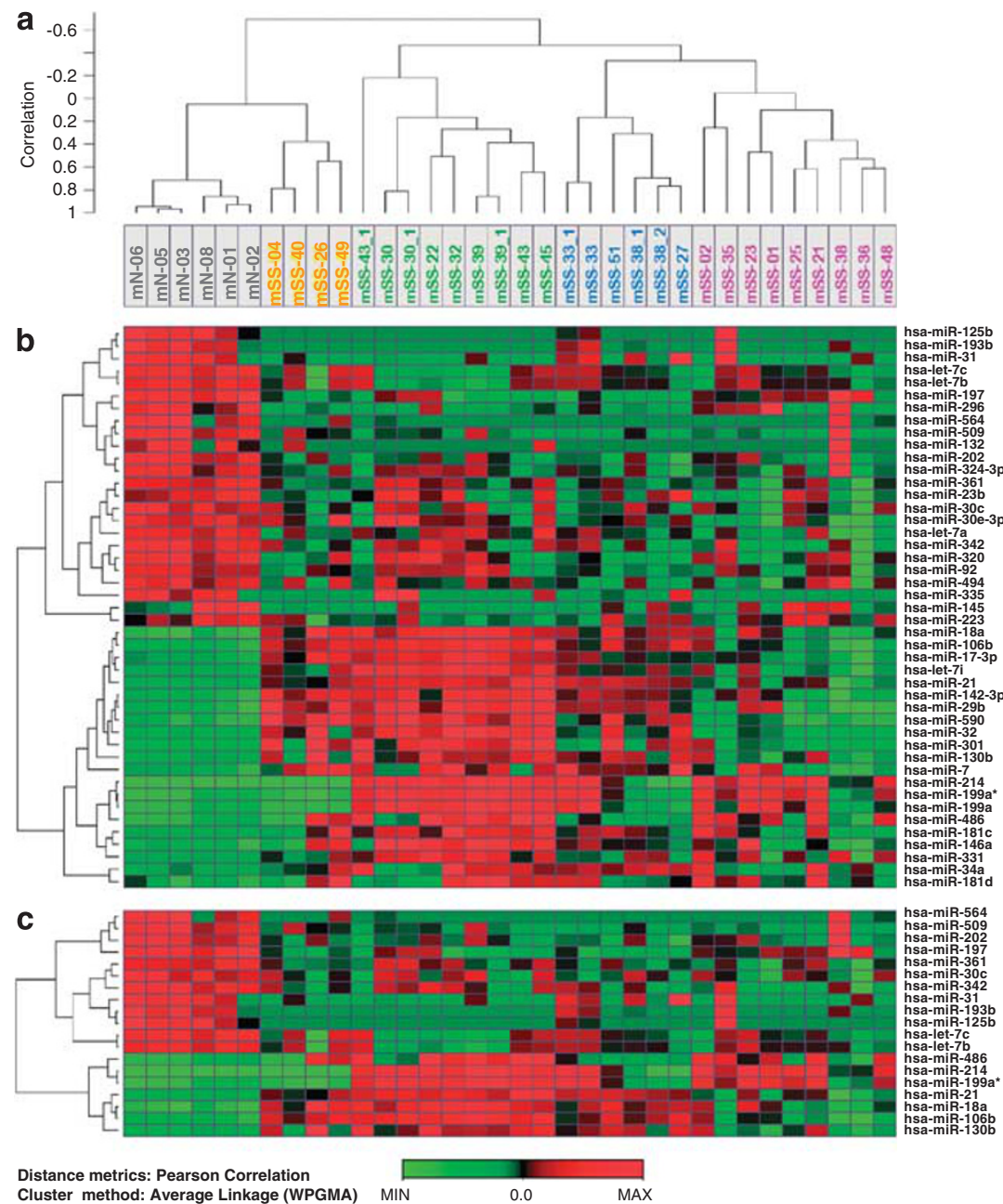

d

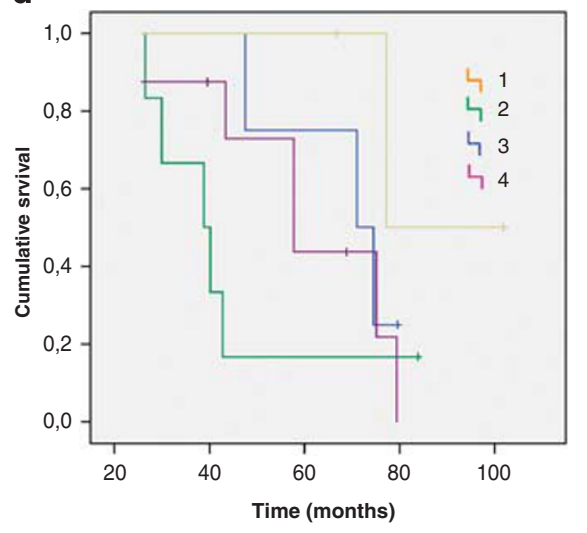

e

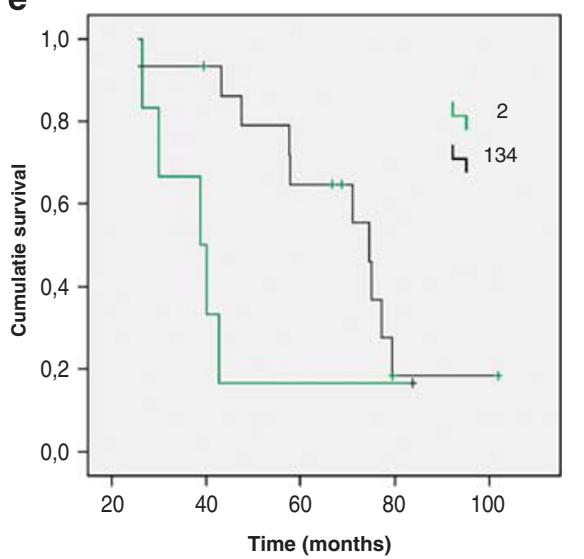

Figure 1 Analysis of miRNA expression profiles and survival curves in the SS cohort hierarchical clustering of the samples, showing the most significant miRNAs $(P \leq 0.01)$ (patients in columns, miRNAs in rows). (a) Dendrogram obtained using Pearson centered correlation distance metric and average linkage cluster method (for the correlated heat map see Supplementary Figure S1). (b) Heat map of the 45 miRNAs differentially expressed between SS and HC. (c) Heat map of predictive analysis between $\mathrm{HC}$ and SS miRNAs selected by class prediction analysis. (d) KM analysis performed on four clusters of SS patients identified by unsupervised hierarchical cluster analysis showing the corresponding MS time (Wilcoxon test $P=0.047$ ). (e) KM analysis performed with cluster $n .2$ (green) versus cluster n. 1, 3 and 4 showing an MS time of 38.84 and 74.57 months, respectively (Wilcoxon test $P=0.011$ )

occurred in these patients. Statistical analysis performed to validate the four subgroups of SS patients is shown in Supplementary Table S2.

A class comparison analysis between HCs and SS lead us to identify the 45 most deregulated miRNAs $(P \leq 0.01)$ able to discriminate the two groups; 21 miRNAs were upregulated and 24 downregulated. They are displayed in the heat map of Figure $1 \mathrm{~b}$ and listed in Table 1.

By using a prediction analysis of BRB array tools (see Materials and Methods), we identified an additional list of 19 miRNAs able to predict in which class (SS or $\mathrm{HC}$ ) one sample could be enclosed relatively to its expression profile (Figure 1c and Supplementary Table S3). This list included miRNAs already highlighted among the 45 most deregulated miRNAs. Most of them were reported to function either as oncogenes like miR-21, ${ }^{14}$ miR-214, ${ }^{15}$ miR- $486{ }^{16}$ and miR-18a belonging to miR-17-92 cluster, or tumor suppressors like let- 7 members and miR-31. ${ }^{17}$
miRNA expression profiling delineates also survival classes in SS. We next sought to determine whether the four groups of SS samples identified by unsupervised analysis were associated with prognosis. As clinical data, including those regarding the survival, were available for the SS patients enrolled in this study (see Supplementary Table S1), we used these four SS clusters to evaluate their survival by Kaplan-Meier (KM) estimator. As shown in Figure 1d, we found that four groups of SS patients displayed four different survival curves, resulting in a statistical correlation (Wilcoxon test $P=0.047$ ) with a median for survival (MS) time of 71 , 38.8, 77 and 57.7 months, respectively (compare matching colors in Figures $1 \mathrm{a}$ and d). These data indicate that cluster n.1 (yellow), the closest one to normal controls, and cluster n.3 (blue) represented the patients with longer survival (MS of 77 and 71, respectively), followed by cluster n.4 (violet) with a MS of 57.73. Conversely, cluster n.2 (green) showed a marked survival decrease with an MS of 38.84 (Figure 1d). 
Table 1 The 45 most significant miRNAs $(P<0.01)$ identified by class comparison analysis performed between $\mathrm{HCs}$ an $\mathrm{SS}$

\begin{tabular}{|c|c|c|c|c|}
\hline Unique id & $\begin{array}{l}\text { Chromosomal } \\
\text { location }\end{array}$ & $\begin{array}{l}\text { Fold- } \\
\text { change }\end{array}$ & FDR & $\begin{array}{l}\text { Parametric } \\
P \text {-value }\end{array}$ \\
\hline hsa-miR-214 & $1 q 24.3$ & 51.78 & 0.00214 & 1.02E-04 \\
\hline hsa-miR-199a* & $19 \mathrm{p} 13.2$ & 37.85 & 0.00343 & $2.76 \mathrm{E}-04$ \\
\hline hsa-miR-199a & $1 \mathrm{q} 24.3$ & 10.27 & 0.00898 & $1.55 \mathrm{E}-03$ \\
\hline hsa-miR-142-3p & $17 q 22+$ & 5.19 & 0.00357 & 3.35E-04 \\
\hline hsa-miR-486 & 8p11.21+ & 5.08 & 0.00398 & $4.81 \mathrm{E}-04$ \\
\hline hsa-miR-29b & $7 q 32.3$ & 4.83 & 0.00898 & $1.57 \mathrm{E}-03$ \\
\hline hsa-miR-146a & $5 q 33.3$ & 4.46 & 0.00898 & $1.38 \mathrm{E}-03$ \\
\hline hsa-miR-34a & $1 \mathrm{p} 36.22$ & 4.23 & 0.00995 & $1.86 \mathrm{E}-03$ \\
\hline hsa-miR-18a & $13 q 31.3$ & 4.12 & 0.00034 & $4.60 \mathrm{E}-06$ \\
\hline hsa-miR-21 & $17 q 23.1+$ & 2.65 & 0.00995 & $1.87 \mathrm{E}-03$ \\
\hline hsa-miR-590 & $7 q 11.23$ & 2.62 & 0.00898 & $1.55 \mathrm{E}-03$ \\
\hline hsa-miR-106b & $7 q 22.1$ & 2.41 & 0.00357 & $3.21 \mathrm{E}-04$ \\
\hline hsa-miR-32 & $9 q 31.3-$ & 2.30 & 0.01507 & $3.64 \mathrm{E}-03$ \\
\hline hsa-miR-181c & $19 p 13.13$ & 2.13 & 0.00696 & 8.87E-04 \\
\hline hsa-miR-17-3p & $13 q 31.3$ & 2.08 & 0.01358 & 2.82E-03 \\
\hline hsa-let-7i & $12 q 12$ & 2.05 & 0.01358 & $2.83 \mathrm{E}-03$ \\
\hline hsa-miR-7 & $\begin{array}{l}9 \mathrm{p} 21.32 / \\
15 q 26.1 / \\
19 p 13.3\end{array}$ & 2.04 & 0.01358 & $2.66 \mathrm{E}-03$ \\
\hline hsa-miR-301 & $17 q 22+$ & 1.95 & 0.01478 & 3.47E-03 \\
\hline hsa-miR-130b & $22 \mathrm{q} 11.21$ & 1.88 & 0.01739 & $4.55 \mathrm{E}-03$ \\
\hline hsa-miR-181d & $19 p 13.13$ & 1.82 & 0.01478 & 3.47E-03 \\
\hline hsa-miR-331 & $12 q 22$ & 1.68 & 0.02152 & $6.21 \mathrm{E}-03$ \\
\hline hsa-miR-132 & 17p13.3- & -1.35 & 0.00343 & $2.70 \mathrm{E}-04$ \\
\hline hsa-miR-564 & 3p21.31 & -1.37 & 0.00103 & 3.46E-05 \\
\hline hsa-miR-335 & $7 q 32.2$ & -1.42 & 0.01474 & $3.17 \mathrm{E}-03$ \\
\hline hsa-miR-296 & $20 q 13.32$ & -1.48 & 0.01587 & $3.96 \mathrm{E}-03$ \\
\hline hsa-miR-30e-3p & $1 \mathrm{q} 34.2$ & -1.51 & 0.02491 & 7.36E-03 \\
\hline hsa-miR-324-3p & $7 \mathrm{p} 13.1$ & -1.59 & 0.02152 & $5.86 \mathrm{E}-03$ \\
\hline hsa-miR-92 & $13 q 31.3$ & -1.66 & 0.03171 & $9.58 \mathrm{E}-03$ \\
\hline hsa-miR-23b & $9 q 22.32$ & -1.70 & 0.01587 & 4.05E-03 \\
\hline hsa-miR-30c & $1 \mathrm{p} 34.2 / 6 \mathrm{q} 13$ & -1.74 & 0.00398 & $4.38 \mathrm{E}-04$ \\
\hline hsa-miR-494 & $14 q 32.31$ & -1.75 & 0.01476 & 3.27E-03 \\
\hline hsa-miR-509 & Xq27.3 & -1.77 & 0.00214 & $9.43 \mathrm{E}-05$ \\
\hline hsa-miR-202 & $10 q 26.3-$ & -1.80 & 0.00698 & $9.84 \mathrm{E}-04$ \\
\hline hsa-let-7a & 9q22.32- & -1.86 & 0.00698 & $9.78 \mathrm{E}-04$ \\
\hline hsa-miR-320 & 8p21.3+ & -1.90 & 0.00898 & $1.47 \mathrm{E}-03$ \\
\hline hsa-miR-361 & Xq21.2 & -1.96 & 0.00214 & $1.19 \mathrm{E}-04$ \\
\hline hsa-miR-197 & $1 \mathrm{p} 13.3$ & -2.08 & 0.00101 & 2.61E-05 \\
\hline hsa-miR-342 & $12 q 22$ & -2.26 & 0.00398 & $4.71 \mathrm{E}-04$ \\
\hline hsa-miR-31 & 9p21.3- & -2.30 & 0.02152 & $6.12 \mathrm{E}-03$ \\
\hline hsa-miR-193b & $16 p 13.12$ & -2.30 & 0.00214 & $1.29 \mathrm{E}-04$ \\
\hline hsa-miR-145 & $5 q 32$ & -2.48 & 0.00369 & $3.71 \mathrm{E}-04$ \\
\hline hsa-miR-125b & $11 q 24.1$ & -2.49 & 0.00034 & $2.90 \mathrm{E}-06$ \\
\hline hsa-miR-223 & Xq12 & -2.69 & 0.02152 & $6.03 E-03$ \\
\hline hsa-let-7c & $21 \mathrm{q} 21.1$ & -3.04 & 0.00101 & $2.70 \mathrm{E}-05$ \\
\hline hsa-let-7b & $22 q 13.31$ & -3.27 & 0.00343 & 2.31E-04 \\
\hline
\end{tabular}

Abbreviations: HCs, healthy controls; miRNA, microRNA; SS, Sézary syndrome.

In bold are indicated chromosomal regions involved in gains $(+)$ and losses $(-)$ in SS.

To increase class numbers and simplify data analysis, we compared the survival time of SS patients belonging just to cluster n.2 (green) with all other patients. The resulting KM analysis delineated the cluster n.2 as the group of SS patients with the worst outcome, showing an MS of 38.84 versus a group with MS of $74.57(P=0.011)$ (Figure 1e).

We also asked which miRNAs could discriminate these two different survival groups. For this reason, we performed a class comparison between the patients belonging to cluster n.2 (mSS n.43, 30, 22, 32, 39 and 45) versus all other patients (clusters 1, 3 and 4). In this way, we identified a list of 47 miRNAs, all upregulated in individuals with the worse outcome (Table 2).

As we observed a significant association between miRNA expression profiles and SS survival, we tried to establish if a
Table 2 miRNAs differentially expressed between SS patients with unfavorable and favorable outcome

\begin{tabular}{|c|c|c|c|c|}
\hline Unique id & $\begin{array}{l}\text { Chromosomal } \\
\text { location }\end{array}$ & $\begin{array}{l}\text { Fold- } \\
\text { change }\end{array}$ & FDR & $\begin{array}{l}\text { Parametric } \\
\text { p-value }\end{array}$ \\
\hline hsa-miR-199a* & $19 p 13.2$ & 18.39 & 6.42E-03 & 8.08E-04 \\
\hline hsa-miR-214 & $1 q 24.3$ & 13.70 & 1.21E-02 & 3.56E-03 \\
\hline hsa-miR-199a & $1 q 24.3$ & 11.33 & 3.78E-03 & 1.40E-04 \\
\hline hsa-miR-146a & $5 q 33.3$ & 4.30 & 5.60E-03 & 3.07E-04 \\
\hline hsa-miR-29b & $7 q 32.3$ & 4.01 & 1.08E-02 & 1.90E-03 \\
\hline hsa-miR-142-3p & $17 q 22+$ & 3.66 & 7.96E-03 & 1.17E-03 \\
\hline hsa-miR-486 & 8p11.21+ & 3.64 & 1.06E-02 & 1.73E-03 \\
\hline hsa-miR-155 & 21q21.2 & 3.30 & 5.60E-03 & 3.75E-04 \\
\hline hsa-miR-30e-5p & $1 q 34.2$ & 3.12 & 1.11E-02 & 2.21E-03 \\
\hline hsa-miR-101 & 1p31.3 & 2.97 & 1.21E-02 & 3.45E-03 \\
\hline hsa-miR-32 & $9 q 31.3-$ & 2.69 & 2.37E-03 & 7.03E-05 \\
\hline hsa-miR-142-5p & $17 q 22+$ & 2.60 & 5.81E-03 & 5.59E-04 \\
\hline hsa-let-7i & $12 q 12$ & 2.58 & 1.35E-04 & 1.00E-06 \\
\hline hsa-miR-21 & 17q23.1+ & 2.56 & 5.81E-03 & $6.51 \mathrm{E}-04$ \\
\hline hsa-miR-29a & $7 q 33$ & 2.39 & 7.03E-03 & 9.38E-04 \\
\hline hsa-miR-374 & Xq21.1 & 2.33 & 1.43E-02 & 4.99E-03 \\
\hline hsa-miR-590 & $7 q 11.23$ & 2.21 & $1.31 \mathrm{E}-02$ & 4.36E-03 \\
\hline hsa-miR-192 & $11 q 13.1$ & 2.13 & 1.11E-02 & 2.14E-03 \\
\hline hsa-miR-17-3p & $13 q 31.3$ & 2.11 & 5.60E-03 & 4.52E-04 \\
\hline hsa-miR-106b & $7 q 22.1$ & 2.09 & 5.60E-03 & 3.96E-04 \\
\hline hsa-miR-16 & $13 q 31$ & 2.08 & 7.99E-03 & 1.24E-03 \\
\hline hsa-miR-20a & $13 q 31.3$ & 2.06 & 1.20E-02 & 2.86E-03 \\
\hline hsa-miR-26a & $3 p 22.2$ & 2.05 & 1.22E-02 & 3.69E-03 \\
\hline hsa-let-7g & $3 p 21$ & 2.01 & $1.24 \mathrm{E}-02$ & 3.86E-03 \\
\hline hsa-miR-18a & $13 q 31.3$ & 2.00 & 1.43E-02 & 4.96E-03 \\
\hline hsa-miR150 & $19 q 13.33$ & 1.97 & 1.20E-02 & 2.76E-03 \\
\hline hsa-miR210 & $11 p 15.5$ & 1.94 & 6.57E-04 & $1.46 \mathrm{E}-05$ \\
\hline hsa-let-7f & 9q22.32- & 1.93 & 1.20E-02 & 2.94E-05 \\
\hline hsa-let-7e & $19 q 13.41$ & 1.90 & 5.81E-03 & 6.73E-04 \\
\hline hsa-miR-34b & $11 q 23.1$ & 1.89 & 1.31E-02 & 4.36E-03 \\
\hline hsa-miR-301 & 17q22+ & 1.86 & 1.20E-02 & 2.89E-03 \\
\hline hsa-miR-194 & $1 q 41 / 11 q 13.1$ & 1.83 & 1.20E-02 & 2.65E-03 \\
\hline hsa-miR-195 & 17p13.1- & 1.82 & 5.81E-03 & 6.89E-04 \\
\hline hsa-miR-181c & $19 p 13.13$ & 1.81 & 1.31E-02 & 4.31E-03 \\
\hline hsa-miR-181c & $19 p 13.13$ & 1.80 & 5.60E-03 & 4.57E-04 \\
\hline hsa-miR-28 & $3 q 28$ & 1.76 & 1.21E-02 & 3.25E-03 \\
\hline hsa-miR-107 & $10 q 22.31-$ & 1.74 & 5.81E-03 & 6.51E-04 \\
\hline hsa-miR-185 & $22 q 11.21$ & 1.71 & 1.08E-02 & 2,00E-903 \\
\hline hsa-miR-30b & 8q24.22+ & 1.71 & 1.21E-02 & 3.04E-03 \\
\hline hsa-miR-25 & $7 q 22.1$ & 1.70 & 5.60E-03 & 3.42E-04 \\
\hline hsa-miR-331 & $12 q 22$ & 1.69 & $1.21 \mathrm{E}-02$ & 3.30E-03 \\
\hline hsa-let-7d & 9q22.32- & 1.61 & 1.08E-02 & 1.96E-03 \\
\hline hsa-miR-215 & $1 q 41$ & 1.58 & 7.96E-03 & 1.18E-03 \\
\hline hsa-miR-30d & 8q24.22+ & 1.57 & 1.20E-02 & $2.75 \mathrm{E}-03$ \\
\hline hsa-miR-191 & $3 p 21.31$ & 1.56 & 1.21E-02 & 3.40E-03 \\
\hline hsa-miR-148b & $12 q 13.13$ & 1.53 & 1.21E-02 & 3.60E-03 \\
\hline hsa-miR-769-5p & $19 q 13.32$ & 1.40 & 1.96E-04 & 2.90E-06 \\
\hline
\end{tabular}

Abbreviations: miRNA, microRNA; SS, Sézary syndrome.

In bold are indicated chromosomal regions involved in gains (+) and losses (-) in SS.

limited number of miRNAs might be used to build a predictive model for SS clinical outcome. To identify the most predictive miRNAs, we used the survival risk prediction algorithm implemented in BRB-ArrayTools ${ }^{18}$ on SS samples described above. The methodological principles of this algorithm have been described. ${ }^{19}$ In brief, high- and a low-risk survival groups were defined by a multivariate model based on gene expression levels contained in each gene signature, the Cox regression coefficient for each gene (supervised principal component method) and two covariates as sex and age.This multivariate model was used in a leave-one-out cross validation process to assign risk-group membership for clinical samples. Statistical significance of the survival groups was assessed by the log-rank test. The top-ranking miRNAs 
Table 3 Survival risk prediction analysis based on miRNA expression (a) and age and sex covariates (b)

\begin{tabular}{|c|c|c|c|}
\hline miRNA ID ${ }^{a}$ & $\begin{array}{l}\text { Chromosome } \\
\text { location }\end{array}$ & $\begin{array}{c}\text { \% CV } \\
\text { Support }\end{array}$ & $P$-value \\
\hline hsa-miR-30d & $8 q 24.2$ & 100 & 0.0004324 \\
\hline hsa-miR-486 & $8 p 11.21$ & 100 & 0.0007376 \\
\hline hsa-let-7d & $9 q 22.32$ & 100 & 0.0010049 \\
\hline hsa-miR-454-3p & $17 q 22$ & 100 & 0.0018857 \\
\hline hsa-miR-93 & $7 q 22.1$ & 100 & 0.0024554 \\
\hline hsa-miR-15b & $3 q 25.33$ & 100 & 0.0050818 \\
\hline hsa-miR-155 & $21 q 21.2$ & 100 & 0.0057033 \\
\hline hsa-miR-16 & $13 q 14.2$ & 100 & 0.0071815 \\
\hline hsa-miR-21 & $17 q 23.1$ & 95.24 & 0.0101705 \\
\hline hsa-miR-31 & $9 p 21.3$ & 95.24 & 0.0142497 \\
\hline hsa-miR-107 & $10 q 23.31$ & 100 & 0.015934 \\
\hline hsa-miR-422b & $5 q 33.1$ & 95.24 & 0.0213468 \\
\hline hsa-miR-15a & $13 q 14.2$ & 90.48 & 0.0221395 \\
\hline hsa-miR-142-5p & $17 q 22$ & 90.48 & 0.0251359 \\
\hline hsa-miR-18a & $13 q 31.3$ & 85.71 & 0.0263155 \\
\hline hsa-let-7a & $9 q 22.32$ & 80.95 & 0.0272317 \\
\hline hsa-miR-301 & $17 q 22$ & 90.48 & 0.0274629 \\
\hline hsa-miR-34b & $11 q 23$ & 90.48 & 0.0281414 \\
\hline hsa-let-7i & $12 q 14.1$ & 71.43 & 0.029799 \\
\hline hsa-miR-181c & $19 p 13.13$ & 57.14 & 0.0392069 \\
\hline hsa-miR-33 & $22 q 13.2$ & 42.86 & 0.0410688 \\
\hline hsa-let-7f & $9 q 22.31$ & 57.14 & 0.0433045 \\
\hline hsa-miR-17-3p & $13 q 31.3$ & 47.62 & 0.0445715 \\
\hline
\end{tabular}

(b)

\begin{tabular}{|c|c|c|c|}
\hline Sample & $\begin{array}{l}\text { Survival time } \\
\text { (months) }\end{array}$ & $\begin{array}{l}\text { Censoring } \\
\text { indicator } \\
(0=\text { alive, } \\
1=\text { dead })\end{array}$ & $\begin{array}{l}\text { Predicted } \\
\text { risk }\end{array}$ \\
\hline mSS-04 & 54 & 1 & High \\
\hline mSS-40 & 81 & 0 & Low \\
\hline mSS-26 & 71 & 1 & High \\
\hline mSS-49 & 74 & 1 & Low \\
\hline mSS-30 & 88 & 0 & Low \\
\hline mSS-22 & 30 & 1 & High \\
\hline mSS-32 & 40 & 1 & High \\
\hline mSS-39 & 26 & 1 & High \\
\hline mSS-43 & 38 & 1 & High \\
\hline mSS-45 & 42 & 1 & High \\
\hline mSS-33 & 66 & 0 & Low \\
\hline mSS-51 & 68 & 1 & High \\
\hline mSS-27 & 174 & 1 & Low \\
\hline mSS-02 & 75 & 1 & Low \\
\hline mSS-23 & 79 & 1 & Low \\
\hline mSS-01 & 90 & 1 & Low \\
\hline mSS-25 & 26 & 1 & High \\
\hline mSS-21 & 44 & 1 & High \\
\hline mSS-38 & 101 & 0 & Low \\
\hline mSS-36 & 72 & 0 & Low \\
\hline mSS-48 & 48 & 1 & High \\
\hline
\end{tabular}

Abbreviation: miRNA, microRNA.

aList of 23 genes selected by fitting Cox proportional hazards models to be the best risk classifiers (alpha $=0.05$ ).

In bold are indicated miRNAs mapping on chromosomal regionsof gain or loss.

selected by this analysis were $23(P<0.042)$. Among them, miR-30d and miR-486 appeared the most significantly riskassociated $(P \leq 0.0007)$ (Table $3 a)$. In good agreement with our previous KM survival analyses (Figures 1d and e), we observed that five out six SS patients (83\%) showing poor outcome were classified as high-risk patients using this prediction model. Moreover, we obtained a consistent classification into low-risk group for $9 / 15$ SS patients $(60 \%)$ previously associated with a favorable outcome (Figures 1a and d). The misclassification of 4/7 (mSS 48, 21, 25 and 51) remaining patients might be explained by the moderate risk of disease observed for these individuals (Figures 1a and d), as also indicated by their intermediate survival time showed in Table $3 \mathrm{~b}$. These data obviously require further validations in independent sets of samples in order to develop an accurate and unbiased classification profile that might be used to predict at which risk-class a future patient will be associated.

With the aim to further restrict the list of prognostic miRNAs, we also intersected the list of the 47 miRNAs showed in Table 2 and the list of 23 miRNAs obtained by Risk prediction analysis (Table 3a). This approach highlighted 14 common miRNAs, such as let-7d, let-7f, let-7i, miR-107, miR-142-5p, miR-155, miR-16, miR17-3p, miR-181c, miR-18a, miR-21, miR-301, miR-30d and miR-486 (Figure 2).

Validation of the miRNA signatures by qRT-PCR. To confirm the miRNA array data, we performed a qRT-PCR using CD4 + purified from PBMCs. We investigated the expression of miR-21, miR-214 and miR-486 on a total of 23 samples, of which 11 were new cases (samples SS41 and from SS52 to SS 64). Of these, 10 are alive (24-36 months follow-up), whereas SS63 has 68 months of survival. (Figures 3a-c). We also validated miR-18a, miR-342, let7b and let7c in three HCs and nine SS patients (Supplementary Figure S2).

MiR-21 (Figure 3a) showed upregulation in 10 out of 21 samples (48\%) (with an FC ranging from 1.4 to 4$)(P<0.03)$. Interestingly, it resulted overexpressed (with an FC ranging from 1.4 to 3 ) in five of five patients (100\%) associated with a poor prognosis (mSS 30, 32, 39, 43 and 45), and at levels comparable to healthy donors in six of seven patients $(86 \%)$ associated with a better outcome. These findings seem to indicate miR-21 as a more strictly potential prognostic marker in SS. In addition, as miR-21 maps at chromosome 17q23, a region frequently found amplified in $S S,{ }^{6}$ we tried to correlate the miR-21 expression with gains on 17q23 (highlighted with black bars, in Figure 3a), but we did not observe any significant correlation. miR-214 resulted over-expressed (with an FC ranging from 2 to 2000) in 17 of 22 cases (73\%) $(P \leq 0.004)$, whereas miR-486 was upregulated (with an $\mathrm{FC}$ ranging 1.8 to 29$)$ in 18 of 23 patients $(83 \%)(P \leq 0.0038)$. These data suggest that both these miRs represent useful diagnostic markers for SS. Moreover, interestingly, miR-214 resulted absent or at very low level (comparable to $\mathrm{HCs}$ ) in five of six long survivors (SS26, 36, 40, 51, 59 and 63) analyzed by qRT-PCR, suggesting its active role in SS pathogenesis.

MiR-21, miR-214 and miR-486 promote cell survival in Hut78 cell line. Because miR-21, miR-214 and miR-486 overexpression were identified in this study as predictive of SS disease and associated to an unfavorable outcome, we investigated the potential effect on apoptosis and cell survival on the HUT78 CTCL cell line by modulating their expression. Hut78 shows miR-21 expression levels comparable to $\mathrm{HCs}$, whereas it does not express miR-214 and miR-486 (Figure 3). For these reasons, we followed an miR-21 lossof-function and an miR-214 and miR-486 gain-of-function approaches. For miR-21 analysis, we transfected cells with 


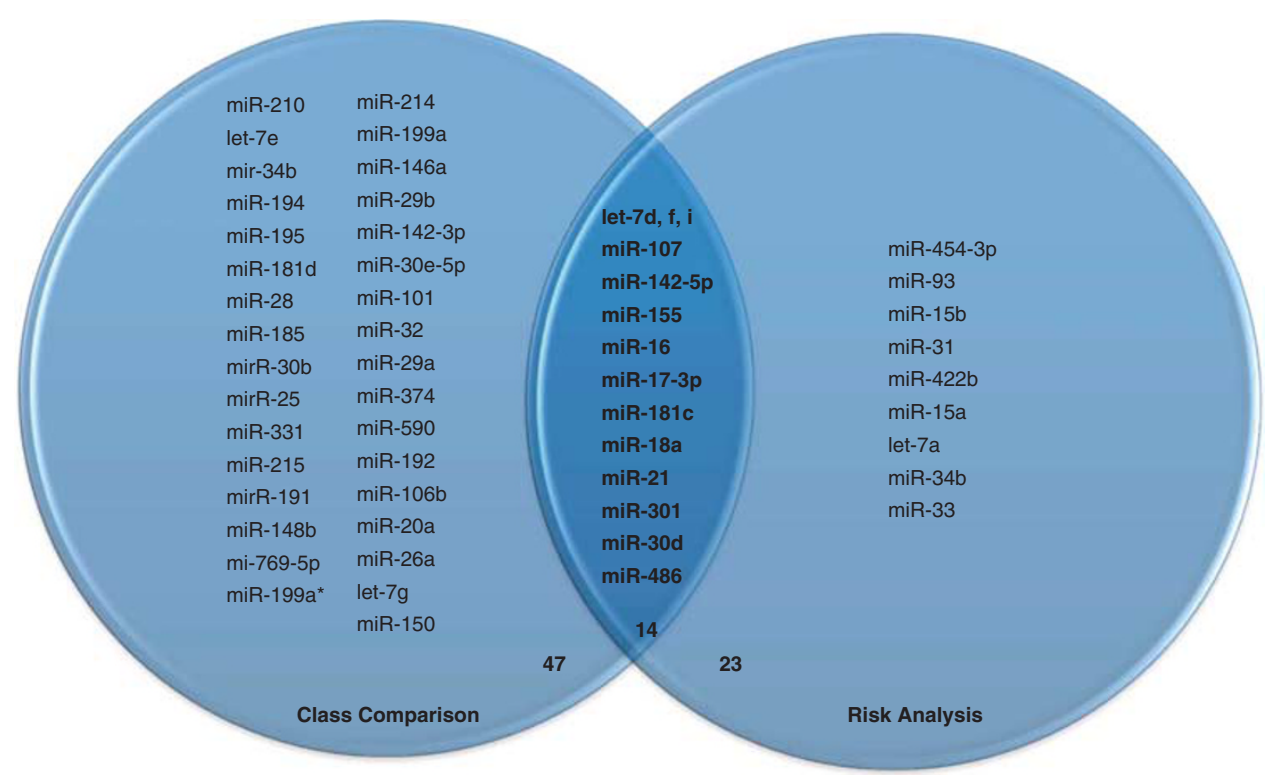

Figure 2 Intersection of two independent prognostic miRNA profiles. A total of 14 common miRNAs were identified intersecting miRNAs found by class comparison performed between patients with the worst and better outcome ( 47 miRNAs) and risk analysis (23 miRNAs)
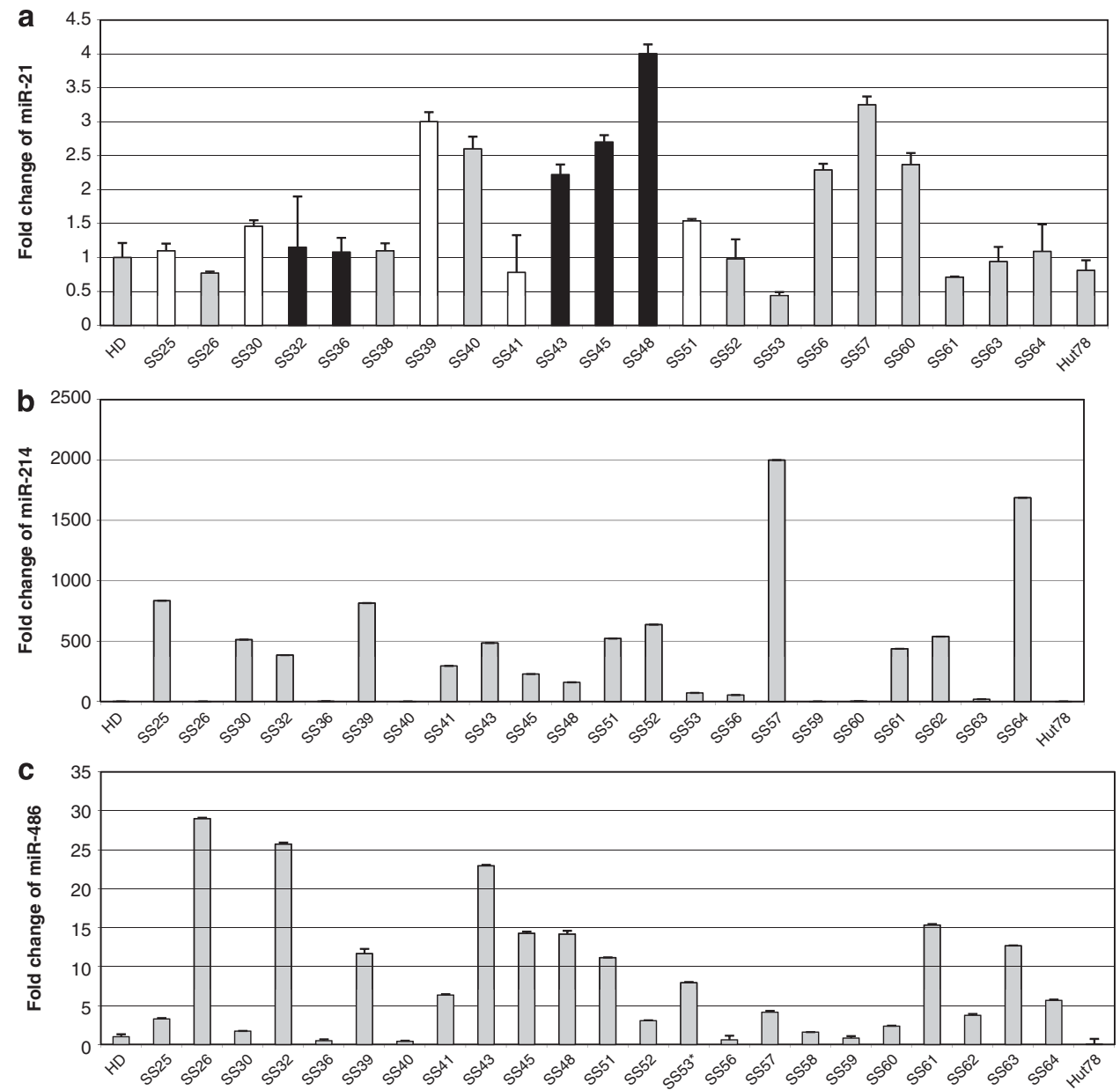

Figure 3 qRT-PCR validation of miR-21 (a), miR-214 and (b) miR-486 (c). miRNA level was calculated for each SS pz relatively to a reference pool constituted by three HDs. In a, Black bars represented patients with a gain of $17 \mathrm{q} 23$ region, whereas white bars represented patients with normal ch $17 q 23$ region. In gray are showed all other samples that were not analyzed by SNPs or CGH arrays 

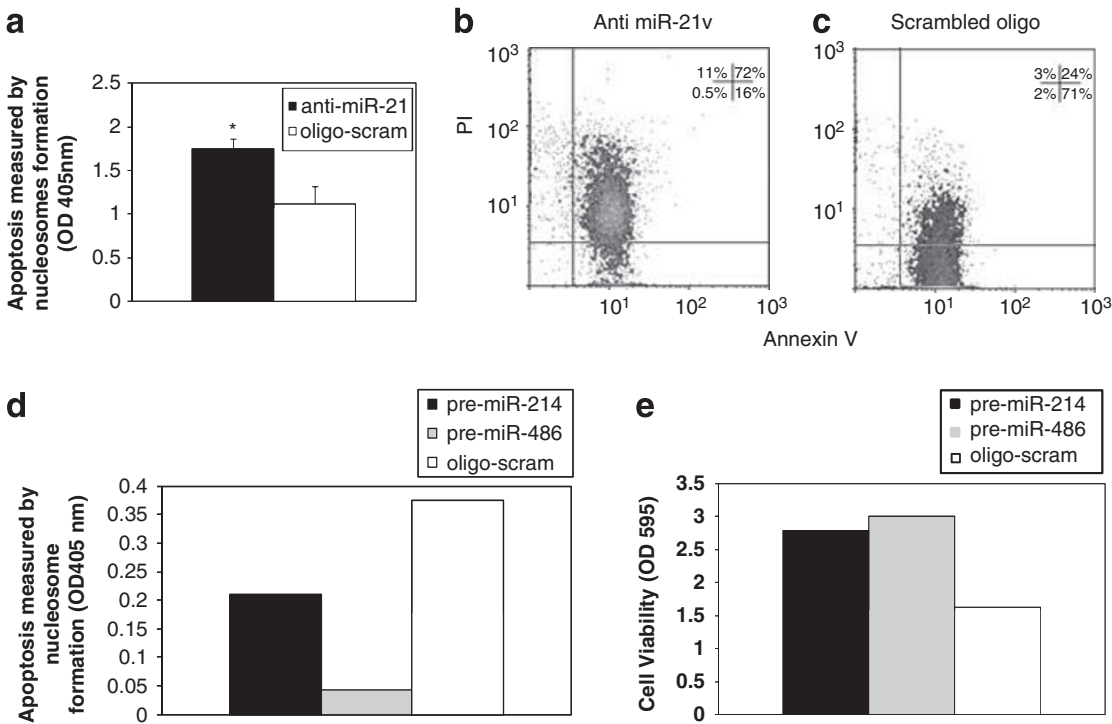

Figure 4 miR-21, miR-214 and miR-486 increase apoptotic resistance in Hut78 cell line. (a) Apoptosis measured by nucleosome formation of HUT78 cells transfected with anti-miR21 and scrambled oligo, used as control. (b and $\mathbf{c}$ ) Apoptosis assessed by flow cytometry $48 \mathrm{~h}$ post-transfection. Anti-miR-21 transfected cells showed a significant increase of apoptotic Annexin V + $/ \mathrm{PI}+$ cells compared with control. (d) Apoptosis measured by nucleosome formation of HUT78 cells transfected with pre-miR-214 and premiR-486 compared with control (e) MTT assay showing increased cell viability of Hut 78 cells transfected with pre-miR-214 and pre-miR-486, compared with control. ${ }^{*} P<0.05$.

anti-miR-21 LNA-knockdown probe and a control-scrambled oligonucleotide to assess the biological effects $48 \mathrm{~h}$ posttransfection $(n=6)$. Transfection efficiency was $>90 \%$ as determined by FACS analysis, and the resulting miR-21 modulation was assessed by qRT-PCR (Supplementary Figure S3). MiR-21 knockdown significantly enhanced the apoptosis in transfected cells compared with controls as determined by the quantification of cytoplasmic histoneassociated DNA fragments (Figure $4 \mathrm{a}$ ) and by Annexin V/PI staining (Figures $4 \mathrm{~b}$ and $\mathrm{c}$ ). Conversely we did not observe a significant effect on cell proliferation as determined by MTT assay (not shown).

For miR-214 and miR-486 studies, we transfected cells with pre-miR-214 and pre-miR-486 oligos and at $48 \mathrm{~h}$ posttransfection, we measured apoptosis (Figure 4D) and cell viability by MTT assay (Figure 4 e) $(n=3)$. Results obtained revealed a decreased cell death associated to an increase of cell viability in miR-214 and miR-486 transfected cells compared with control. All these findings indicate that miR-21, miR-214 and miR-486 contribute to the apoptotic resistance of CTCL cell line.

\section{Discussion}

miRNA whole gene analysis has proven to be a valid tool to identify diagnostic and disease progression signatures in several tumors and especially in hematological malignancies. In the present study, we have profiled for miRNA expression $28 \mathrm{SS}$ samples and six $\mathrm{HCs}$, using microarray technology. A total of 45 miRNAs were the most significant among those differentially expressed between SS patients and HCs. Furthermore, we were able to identify 19 miRNAs with a potential ability of disease prediction. Until now, only one study has been done on miRNA expression for SS. ${ }^{13}$ In this investigation, a set of 114 deregulated miRNAs able to distinguish SS from HCs was identified and 20 were the most discriminatory. Our results confirm, in part, these data showing a concordant increased expression of miR-214, miR-199a* and miR-7, along with the decrease of miR-342, miR-223, miR-92, miR-181a and miR-191. Conversely, a discordant expression level was observed for miR-145 and miR-18a. However, we have also found a large fraction of nonoverlapping deregulated miRNAs that might be explained with the different methodological approaches used, such as miRNA platforms, the bioinformatics and statistical methods employed to analyze data and particularly to identify diagnostic and prognostic signatures.

Our unsupervised analysis identified four sub-groups of SS patients and, thus, we wondered whether these different clusters were associated to any clinical parameters such as tumor progression, tumor burden, CD4/CD8 ratio, dominant TCR-V beta rearrangement, genetic lesions and survival. Interestingly, we observed a correlation with clinical outcome, recognizing a group of patients (belonging to cluster n.2) with the shortest survival respect to all other individuals enrolled in this study. We thus compared miRNA expression data with survival time using class comparison and risk prediction analysis. Following this approach, we obtained two independent lists of miRNAs differentially expressed between patients with unfavorable and favorable outcome (Table 1 and 2a). In the first one, miR-214 together with miR-199a and miR$199 \mathrm{a}^{*}$ appeared the most significantly deregulated. The intersection of these two lists, identified 14 miRNAs (Figure 2). Among them, interestingly, we observed again miR-21 and miR-486 that we have studied in detail, miR-155 involved in a variety of tumors ${ }^{20}$ and miR-16 already found deregulated in SS. ${ }^{13}$ Interestingly, many of these potentially prognostic miRNAs map to gain/loss genomic regions frequently found unbalanced in SS, ${ }^{6}$ suggesting that also genomic alterations might contribute to miRNA deregulation in SS.

Several transcriptional or epigenetic mechanisms may influence miRNA changes. Narrowing our attention to the 19 
predictive miRNAs, we noted that miRNA 214/199a cluster and $199 a^{*}$ are co-expressed in our sample set (Figure 1b) and they were previously found to be upregulated by Twist $1 .{ }^{21}$ This is a transcription factor found overexpressed in $S S,{ }^{5}$ so that it might control the upregulation of miR-214/199a/199a* in this lymphoma. As a large fraction of our SS patients were already profiled for gene expression, ${ }^{6}$ we matched miRNA and mRNA results; in this manner, we noted that expression levels of miR-30c correlated with the expression of its host genes NFYC, suggesting a control at level of primary transcription rates. Similarly, miR-342 correlated with its host gene EVL that in turn is downregulated by $199 a^{*} .{ }^{13}$ Moreover, we observed a number of tumor-related miRNAs like miR$18 \mathrm{a}$, belonging to cluster 17-92, and miR-106b (cluster 106b25) strongly involved in lymphoproliferative diseases. ${ }^{9,22}$ Additional members of the same clusters, such as miR-17$3 p$ and miR-18a (cluster 17-92) and miR-93 (cluster 106b25 ), are included in the list of prognostic miRNAs (Table 1 and $2 a)$. The genetic loci encoding the miR-17-92 (13q31) and 106b-25 (7q22) clusters do not appear among the chromosomal region recurrently altered in $S S^{6}{ }^{6}$ therefore, their deregulation should be attributable to other mechanisms. For example, by c-Myc, found overexpressed in SS, ${ }^{5}$ that is able to induce the expression of these miRNAs in several tumors and animal models. ${ }^{23}$

In our dataset, we observed a diminished expression of let$7 a$, let-7b and let-7c (Figure $1 b$ and c). Let-7 family members are widely viewed as tumor suppressor miRNAs and they are strongly downregulated in many cancers and during tumor progression. ${ }^{24}$ Conversely, an upregulation of let-7d, let-7f and let-7i was mainly found in patients with unfavorable prognosis (Table 1 and 2a). A downregulation of miR-31 was observed in 22 out of 28 (79\%) SS patients. miR-31 is an antimetastatic miRNA and its downregulation or deletion of its genomic locus at 9p21 (recurrently observed in SS) promotes invasion/metastasis of cancer. ${ }^{25}$ These findings suggest that, similarly to other cancers, downregulation of miR-31 might contribute to the atypical trafficking ability of these malignant lymphocytes, and might represent another potential diagnostic in SS.

In this study, we focused our attention on miR-21, miR-214 and miR-486, because they most significantly correlated with diagnosis and prognosis of SS. Results indicated that miR214 and miR-486 were over-expressed in the majority of SS patients therefore more likely related to diagnosis, whereas miR-21 upregulation is detected in a smaller number of patients thereby more probably associated to prognosis of SS. Moreover, performing functional experiments, we observed that all these miRs are able to promote cell survival in Hut78 cell line. Interestingly, miR-21 was also recently described to increase survival in another CTCL cell line, ${ }^{26}$ whereas miR-486 and miR-214 have been recently associated to clinical outcome of many tumors. ${ }^{15,16,27,28}$ Moreover, miR-214 over-expression has also been recently described to promote proliferation of healthy $T$ lymphocytes via PTEN targeting. ${ }^{29}$ Among the thousands of miR-21 gene targets, one of the best characterized and involved in neoplastic transformation is PTEN. Thus, it is possible that a concurrent over-expression of miR-21 and miR-214 increases SS cell survival, repressing the PTEN function. In conclusion, our findings indicate that miR-21, miR-214 and miR-486 might represent novel diagnostic/prognostic biomarkers for this incurable variant of CTCL.

\section{Materials and Methods}

Patients and healthy controls. Peripheral blood samples from 22 SS cases, six follow-ups of patients SS30, 33, 38, 39, 43 and six HCs were analyzed. All patients were enrolled in this study approved by the Ethical Committee of the Istituto Dermopatico dell'Immacolata and informed consent was obtained in accordance with the Declaration of Helsinki. Diagnosis of SS was based on described criteria. ${ }^{30}$ The major clinical and immunological characteristics have been reported in Supplementary Table S1 and obtained as described. ${ }^{6}$

miRNA expression profiling. Neoplastic and healthy lymphocytes were purified by dynabeads according to manufacturer's instructions (Invitrogen, Oslo, Norway) and total RNA extracted and quantified as already described in details. ${ }^{6}$ Labeled RNA was then hybridized on Agilent Human V1 miRNA microarray (Agilent Technologies, Santa Clara, CA, USA), consisting of 470 miRNA probes, according to the manufacturer's procedure. Agilent scanner and the Feature Extraction 10.5 software (Agilent Technologies) were used to obtain the microarray raw-data.

Bionformatics analysis. Data analysis was performed, by using customized $\mathrm{R}$ language-based script (www.r-project.org), using the Bioconductor packages (www.bioconductor.org). Quality control analysis, data normalization and hierarchical cluster were performed as described. ${ }^{31}$ Data matrix of normalized values was subjected to class comparison and prediction analysis using BRBArrayTools version $3.9 .0 .^{18}$ Class comparison allowed identifying the miRNAs differentially expressed between SS patients and $\mathrm{HCs}$ using a univariate two-sample $t$-test with a significance threshold of 0.01 , while controlling either the number or proportion of false discoveries. Class prediction was assessed to classify and to predict in which class was enclosed a sample in relation to its expression profile, using multivariate classification methods showed in Supplementary Table S4. Hierarchical clustering of the data was performed with the bioconductor heatmap package, using centered correlation and complete linkage similarity metric. Cluster Stab bioconductor package was used to compute cluster stability scores. In addition, we performed a survival risk prediction using BRB-ArrayTools version 3.9.0. ${ }^{18}$ This tool was used to directly predict the survival risk group based on expression data and two covariates as sex and age.MiR-21 target genes have been predicted applying miR and an algorithm-associated MirBase software (http:// microrna.sanger.ac.uk)

Survival and statistical analyses. A time-to-event analysis was performed using nonparametric KM product-limit survival estimates, and differences between $\mathrm{KM}$ survival curves were analyzed using the Wilcoxon test. Statistical analyses were performed using Statistical Package SPSS v.13.0. Results of qRT-PCR were expressed as mean plus or minus S.D. Statistical analysis was performed using the two-tailed Student $t$-test or one-way ANOVA.

Quantitative real-time PCR (q-RT-PCR). qRT-PCR was used to confirm our microarray data. Selected mature miRNAs were analyzed using TaqMan microRNA assay (Applied Biosystems, Foster City, CA, USA). Z30 was used as an internal normalized reference. Quantitative analysis was performed by the $\triangle C T$ method as described. ${ }^{32}$

Cell line transfections, cell viability and apoptosis. Hut-78 cell line established from peripheral blood of CTCL patient from American Type Culture Collection (TIB161) was grown in RPMI (Sigma Aldrich Biochemicals, St. Louis, MO, USA), supplemented with $10 \%$ heat-inactivated FCS and antibiotics at $37^{\circ} \mathrm{C}$ in a humidified air atmosphere at $5 \% \mathrm{CO}_{2}$. LNA-knockdown miR-21 or LNA-Knockdown probe Scramble molecules $5^{\prime}$ fluorescein (Exiqon, Vedbaek, Denmark), pre-miR-214 and miR-486 or FAM Pre-miR-scrambled negative control (Ambion, Foster City, CA, USA) were transfected using the Amaxa Biosystems nucleofector electroporation system as described ${ }^{33}$ Briefly, $2 \times 10^{6}$ of Hut78 were resuspended in $100 \mu \mathrm{l}$ of nucleofection R, with oligos used at $30 \mathrm{nM}$. After electroporation, the cells were seeded into 24-well plates and the medium was refreshed $\sim 8 \mathrm{~h}$ after transfection. The cell viability and apoptosis were examined $48 \mathrm{~h}$ posttransfection with 3-(4,5-dimethylthiazol-2-yl)-2,5-diphenyltetrazolium bromide (MTT) reduction (Sigma -Aldrich Biochemicals) and quantifying cytoplasmic histone-associated DNA fragments (Roche Molecular Biochemicals, Manneheim, Germany) assays as described. ${ }^{34}$ 
Flow cytometry. Flow cytometric analysis for apoptotic cells was performed by staining cells with FITC-Annexin V and propidium iodide, using the apoptosis detection kit (Clontech, Mountainview, CA, USA) Samples were acquired on a flow cytometer (FacsCalibur, BD Biosciences, San Jose, CA, USA), and analyzed using CellQuest software (BD Biosciences).

\section{Conflict of Interest}

The authors declare no conflict of interest.

Acknowledgements. Grant support. This study has been supported by Ministero della Salute and Associazione Italiana Ricerca sul Cancro (M.G.N.). We thank the nurse staff of III Clinical Dermatology Unit of the IDI-IRCCS for continuous and helpful collaboration. The authors are also grateful to Dr. Debora Pomponi for flow cytometry analyses and Dr. Gianluca Ragone for figures set up.

Authorship: M.G.N. designed the study; M.G.N, C.L., E.P., M.C.P., P.F.C.C, E.S, M.F., A.M. and G.A.L performed research and recruited patients. D.A., M.F., F.S and A.F analysed data. M.G.N., M.N., E.C. and G.R. wrote the paper.

1. Swerdlow S, Campo E, Harris N, Jaffe E, Pileri S, Stein H et al. (eds) WHO Classification of Tumors of Haematopoietic and Lymphoid Tissues. International Agency for Research on Cancer Press: Lyon, France, 2008.

2. Olsen E, Vonderheid E, Pimpinelli N, Willemze R, Kim Y, Knobler R et al. Revisions to the staging and classification of mycosis fungoides and Sezary syndrome: a proposal of the International Society for Cutaneous Lymphomas (ISCL) and the cutaneous lymphoma task force of the European Organization of Research and Treatment of Cancer (EORTC). Blood 2007; 110: 1713-1722.

3. Prince HM, Whittaker S, Hoppe RT. How I treat mycosis fungoides and Sezary syndrome. Blood 2009; 114: 4337-4353.

4. van Doorn R, Dijkman R, Vermeer MH, Out-Luiting JJ, van der Raaij-Helmer EM, Willemze $\mathrm{R}$ et al. Aberrant expression of the tyrosine kinase receptor EphA4 and the transcription factor twist in Sezary syndrome identified by gene expression analysis. Cancer Res 2004; 64: 5578-5586.

5. Vermeer MH, van Doorn R, Dijkman R, Mao X, Whittaker S, van Voorst Vader PC et al. Novel and highly recurrent chromosomal alterations in Sezary syndrome. Cancer Res 2008; 68: 2689-2698.

6. Caprini E, Cristofoletti C, Arcelli D, Fadda P, Citterich MH, Sampogna F et al. Identification of key regions and genes important in the pathogenesis of sezary syndrome by combining genomic and expression microarrays. Cancer Res 2009; 69: 8438-8446.

7. Laharanne E, Oumouhou N, Bonnet F, Carlotti M, Gentil C, Chevret E et al. Genome-wide analysis of cutaneous T-cell lymphomas identifies three clinically relevant classes. J Invest Dermatol 2010; 130: 1707-1718.

8. Carthew RW, Sontheimer EJ. Origins and Mechanisms of miRNAs and siRNAs. Cell 2009; 136: $642-655$

9. Baltimore D, Boldin MP, O'Connell RM, Rao DS, Taganov KD. MicroRNAs: new regulators of immune cell development and function. Nat Immunol 2008; 9: 839-845.

10. Garofalo M, Condorelli GL, Croce CM, Condorelli G. MicroRNAs as regulators of death receptors signaling. Cell Death Differ 2010; 17: 200-208.

11. Melino G, Knight RA. MicroRNAs meet cell death. Cell Death Differ 2010; 17: 189-190.

12. Garzon R, Calin GA, Croce CM. MicroRNAs in Cancer. Annu Rev Med 2009; 60 167-179.

13. Ballabio E, Mitchell T, van Kester MS, Taylor S, Dunlop HM, Chi J et al. MicroRNA expression in Sezary syndrome: identification, function, and diagnostic potential. Blood 2010; 116: 1105-1113

14. Croce CM. Causes and consequences of microRNA dysregulation in cancer. Nat Rev Genet 2009; 10: 704-714.
15. Ueda T, Volinia S, Okumura H, Shimizu M, Taccioli C, Rossi S et al. Relation between microRNA expression and progression and prognosis of gastric cancer: a microRNA expression analysis. Lancet Oncol 2010; 11: 136-146.

16. Hu Z, Chen $X$, Zhao Y, Tian T, Jin G, Shu Y et al. Serum microRNA signatures identified in a genome-wide serum microRNA expression profiling predict survival of non-small-cell lung cancer. J Clin Oncol 2010; 28: 1721-1726.

17. Volinia S, Galasso M, Costinean S, Tagliavini L, Gamberoni G, Drusco A et al. Reprogramming of miRNA networks in cancer and leukemia. Genome Res 2010; 20: 589-599.

18. Simon R, Lam A, Li MC, Ngan M, Menenzes S, Zhao Y. Analysis of Gene Expression Data Using BRB-Array Tools. Cancer Inform 2007; 3: 11-17.

19. Bair E, Tibshirani R. Semi-supervised methods to predict patient survival from gene expression data. PLOS Biol 2004; 2: E108

20. Tili E, Croce CM, Michaille JJ. miR-155: on the crosstalk between inflammation and cancer. Int Rev Immunol 2009; 28: 264-284.

21. Yin G, Chen R, Alvero AB, Fu HH, Holmberg J, Glackin $C$ et al. TWISTing stemness, inflammation and proliferation of epithelial ovarian cancer cells through MIR199A2/214. Oncogene 2010; 29: 3545-3553.

22. Petrocca $F$, Vecchione $A$, Croce CM. Emerging role of miR-106b-25/miR-17-92 clusters in the control of transforming growth factor beta signaling. Cancer Res 2008; 68: 8191-8194.

23. Mu P, Han YC, Betel D, Yao E, Squatrito M, Ogrodowski P et al. Genetic dissection of the miR-17 $\sim 92$ cluster of microRNAs in Myc-induced B-cell lymphomas. Genes Dev 2009; 23: $2806-2811$.

24. Roush S, Slack FJ. The let-7 family of microRNAs. Trends Cell Biol 2008; 18: 505-516.

25. Valastyan S, Reinhardt F, Benaich N, Calogrias D, Szasz AM, Wang ZC et al. A pleiotropically acting microRNA, miR-31, inhibits breast cancer metastasis. Cell 2009; 137 1032-1046.

26. van der Fits L, van Kester MS, Qin Y, Out-Luiting JJ, Smit F, Zoutman WH et al. MicroRNA21 expression in CD4+ T cells is regulated by STAT3 and is pathologically involved in Sezary syndrome. J Invest Dermatol 2011; 131: 762-768.

27. Ragusa M, Majorana A, Statello L, Maugeri M, Salito L, Barbagallo $D$ et al. Specific alterations of microRNA transcriptome and global network structure in colorectal carcinoma after cetuximab treatment. Mol Cancer Ther 2010; 9: 3396-3409.

28. Yang $\mathrm{H}$, Kong $\mathrm{W}$, He L, Zhao JJ, O'Donnell JD, Wang $\mathrm{J}$ et al. MicroRNA expression profiling in human ovarian cancer: miR-214 induces cell survival and cisplatin resistance by targeting PTEN. Cancer Res 2008; 68: 425-433.

29. Jindra PT, Bagley J, Godwin JG, lacomini J. Costimulation-dependent expression microRNA-214 increases the ability of T cells to proliferate by targeting Pten. $J$ Immunol 2010; 185: 990-997.

30. Narducci MG, Scala E, Bresin A, Caprini E, Picchio MC, Remotti D et al. Skin homing of Sezary cells involves SDF-1-CXCR4 signaling and down-regulation of CD26/ dipeptidylpeptidase IV. Blood 2006; 107: 1108-1115.

31. Arcelli D, Farina A, Cappuzzello C, Bresin A, De Sanctis P, Perolo A et al. Identification of circulating placental mRNA in maternal blood of pregnancies affected with fetal congenital heart diseases at the second trimester of pregnancy: implications for early molecular screening. Prenat Diagn 2010; 30: 229-234.

32. Zhang AS, Xiong S, Tsukamoto $\mathrm{H}$, Enns CA. Localization of iron metabolism-related mRNAs in rat liver indicate that HFE is expressed predominantly in hepatocytes. Blood 2004; 103: 1509-1514.

33. Mahmoudi T, Parra M, Vries RG, Kauder SE, Verrijzer CP, Ott M et al. The SWI/SNF chromatin-remodeling complex is a cofactor for Tat transactivation of the HIV promoter. J Biol Chem 2006; 281: 19960-19968.

34. Si ML, Zhu S, Wu H, Lu Z, Wu F, Mo YY. miR-21-mediated tumor growth. Oncogene 2007; 26: 2799-2803

Cell Death and Disease is an open-access journal published by Nature Publishing Group. This work is licensed under the Creative Commons Attribution-Noncommercial-No Derivative Works 3.0 Unported License. To view a copy of this license, visit http://creativecommons.org/licenses/by-nc-nd/3.0/

Supplementary Information accompanies the paper on Cell Death and Disease website (http://www.nature.com/cddis) 